\title{
Editorial
}

\section{Entrepreneurship Education with Impact: Opening the Black Box}

\author{
Thomas Lans, ${ }^{1}$ Päivi Tynjälä, ${ }^{2}$ Harm Biemans, ${ }^{1}$ Tiago Ratinho, ${ }^{3}$ and Saeid Karimi ${ }^{4}$ \\ ${ }^{1}$ Education and Competence Studies Group, Wageningen University \& Research, Hollandseweg 1, 6706 KN Wageningen, Netherlands \\ ${ }^{2}$ Finnish Institute for Educational Research, University of Jyväskylä, P.O. Box 35, 40014 Jyväskylä, Finland \\ ${ }^{3} I E ́ S E G$ School of Management, Paris Campus, 1 parvis de la Défense, Socle de la Grande Arche, La Défense, 92044 Paris, France \\ ${ }^{4}$ Department of Agricultural Extension and Education, Bu-Ali Sina University, Hamedan 65178 33131, Iran \\ Correspondence should be addressed to Thomas Lans; thomas.lans@wur.nl
}

Received 21 September 2017; Accepted 24 September 2017; Published 1 November 2017

Copyright (c) 2017 Thomas Lans et al. This is an open access article distributed under the Creative Commons Attribution License, which permits unrestricted use, distribution, and reproduction in any medium, provided the original work is properly cited.

Entrepreneurship education (EE) has become globally widespread. Governments, universities, and private institutions increasingly deploy resources dedicated to fostering entrepreneurship competencies amongst entrepreneurs as well as in society at large. However, this exponential growth of initiatives did not go hand in hand with a similar development of scholarly research, hence hindering our understanding of the impacts of EE.

Is it nowadays uncontroversial that $\mathrm{EE}$ is more than "just" training entrepreneurs to create more start-ups. In addition, the persistent nature-nurture discussion is moving towards a scholarly consensus that, like in any other profession, talent helps, but there is a lot to be learned to be able to engage in entrepreneurial thinking and acting. At the same time, the common message from EE scholars is that a further research is needed to provide a more scientifically grounded approach to EE in terms of contents and intended outcomes. As several of the articles in this special issue conclude, EE practice seems to be ahead of educational studies, both conceptually and empirically. For instance, entrepreneurship educators have widely adopted in the past decades heuristicsbased approaches to EE such as Ries' Lean Start-up Method, Sarasvathy's effectuation principles, or Osterwalder's Business Model Canvas. However, educational research further investigating these practices from well-known theoretical lenses like problem-based, project-based, or cooperative learning remains scarce.

This promise of EE as well as the work that still needs to be done presents an opportunity for EE scholars. There is a risk that EE will eventually wither amidst the capricious world of educational management, if the field fails to elicit, display, and develop its potential. As the concept of EE remains surrounded with ambiguity, it will remain attractive to many, but when times are changing, it maybe as easily be discarded as well. EE scholars have an important role here to further advance theorizing and to critically assess and inform entrepreneurship educators with evidence-based practices. This means that this field has to move beyond the so-called "intention studies" plain. As we clearly stated in the call for this special issue, there is a clear need to go beyond the current narrow intention models of measuring the impact of EE, as EE is more than a "factory" for creating high-potential start-ups. It is suggested that the impact of EE lies in the development of 21st century skills, such as creativity and complex problem solving, noncognitive skills like social competence and resilience, and even broader fostering employability, identity building, and lifelong learning.

This special issue paves the way for promising streams of research that EE scholars should consider as new lines of research. At the heart of the current challenge in investigating the impact of EE is a need for more systematic approaches to EE. This need is confirmed by U. Kamovich and L. Fross in their contribution to this special issue. Building further on the seminal work of John Biggs, they convincingly show that, over the past 15 years, EE scholars have paid very little attention to constructive alignment. They conclude that reporting about teaching objectives, teaching methods, and teaching content as well as their connection receives scant attention from 
researchers. On one hand, this might be due to editors and reviewers who are not requesting such information from the reported interventions. On the other hand, this could also be due to the fact that entrepreneurship education is not familiar with this well-known concept from educational sciences. One way or another, this systematic review clearly points its finger to one of the backbones of EE research, constructive alignment, in particular when one is interesting in its impact.

The issue of impact and misalignment is further picked up by the review article of $S$. A. Gedeon from the perspective of what quality means for different stakeholders. Based on a thorough investigation of literature on the role of quality from the perspective of accreditation agencies, employers, students, faculties, and award agencies, he concludes that stakeholders have surprisingly divergent opinions on what the goals of EE programs should be. Nonetheless, he argues that putting student learning outcomes (e.g., entrepreneurial competencies) at the heart of discussing the quality of $\mathrm{EE}$ programs helps to harmonize such diverse interests. All other goals and quality aspects of EE programs (like faculty quality and number of start-ups generated) can be considered as input or output factors. As such, S. A. Gedeon questions whether there is indeed lack of rigor or best practices in EE research or that the question for EE scholars lies more in actually tracking "student transformation" in terms of changes in knowledge, skills, and attitudes, that is, competence development.

The issue of competence development is further addressed by U. Lilleväli and $M$. Täks who delved into the value of competence models to systemically investigate student transformation in EE throughout all education levels. As the authors state, "competence models provide a platform to meaningfully embed varying interpretations, learning outcomes and roles of EE, and allow educators and other stakeholders to apply EE systematically throughout all education levels." Thus, competence models can help educational designers, teachers, and learners in the process of systemizing the how, what, and when of teaching certain aspects of EE. Although endless, laundry lists of competencies have already been produced in the last decades; theoretical and empirical works on competence models have largely been ignored. The authors therefore ask themselves the following questions: What do different mapping approaches have in common? Where do they differ? How do they relate specifically to different educational contexts? After investigating five competence models in detail, the authors conclude that these models indeed help in systematizing the EE competence development process and that they also vary significantly amongst the investigated nations and thus cannot blindly be copied. A common feature, however, of all five analysed EE competence models was the focus on developing competencies related to opportunity pursuit, which is not a surprise considering the ample attention to opportunities in the mainstream entrepreneurship literature.

Further advancement of operationalising and measuring competence in educational research is done in an empirical research article by Y. Baggen et al. Although there is considerable effort done in measuring learning outcomes of EE, studies mainly rely on self-assessments: pen-andpencil type of methods which do not cater for the complex and dynamic tasks as they occur during entrepreneurial activities. More specifically, Y. Baggen and colleagues investigate opportunity identification competence in relation to complex problem skill by introducing performance-based assessment methods. The authors further contribute to the relevance of so-called transversal 21st century skills to the domain of EE. The authors conclude that complex problem solving indeed contributes to predicting idea generation of students in higher education. These results emphasize the need to further explore relationships between domain specific constructs from entrepreneurship research and more transversal, generic constructs which have a rich history in mainstream educational psychology literature. Such research will strengthen methodological rigor and will help to collect the necessary empirical evidence on competence development as emphasized in the paper of S. A. Gedeon and the paper of U. Lilleväli and M. Täks.

Finally, S. Jie and R. Harms add to the debate on the impact of EE arguing that internationalisation of higher education represents an international and cross-cultural experience in $\mathrm{EE}$ in a growing number of universities. Cross-cultural competencies should therefore also be considered when talking about desired learning outcomes of EE. Although the results of S. Jie and R. Harms amongst university students do not show a direct influence of cross-culture competence on the intention to engage in activities aimed at starting an international new venture, further analysis does highlight the importance of thoroughly disentangling the relationships between the different constructs we currently use to measure learning outcomes in EE.

Taken together, the set of articles in this special issue helps in establishing EE as an important subfield of entrepreneurship studies. The editors are confident that this selection of articles contributes to the contemporary scholarly debate within the EE field by addressing the unique challenges EE faces and further advancing our understanding of impacts of EE activities.

\author{
Thomas Lans \\ Päivi Tynjälä \\ Harm Biemans \\ Tiago Ratinho \\ Saeid Karimi
}




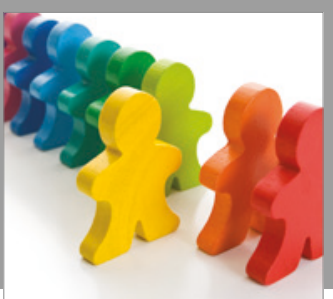

Autism

Research and Treatment
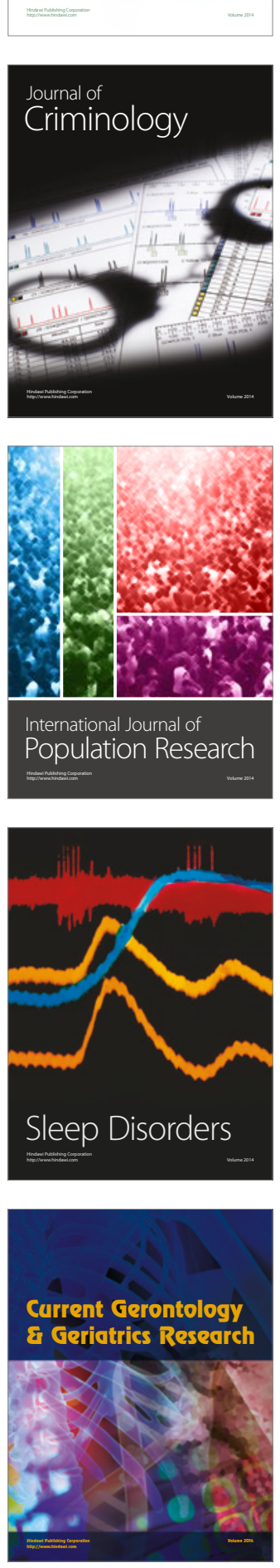

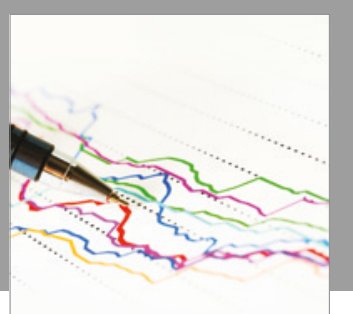

Economics

Research International

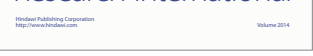

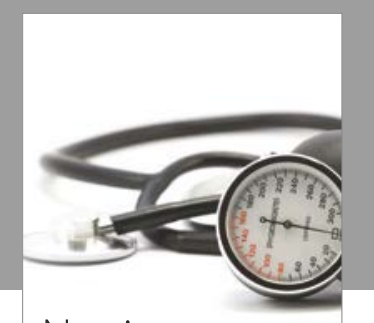

Nursing

Research and Practice

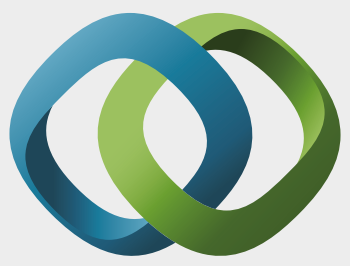

\section{Hindawi}

Submit your manuscripts at

https://www.hindawi.com
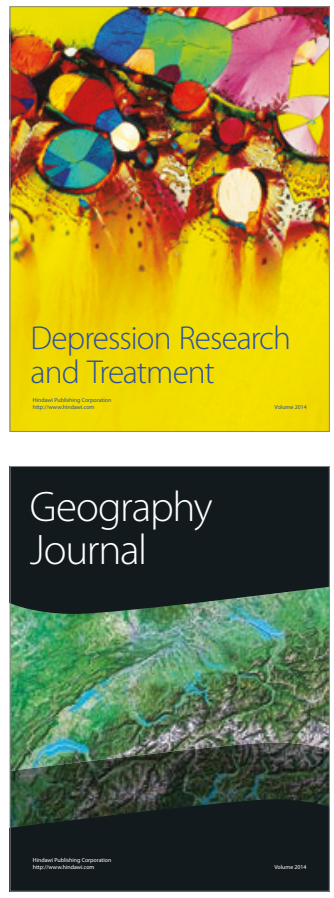
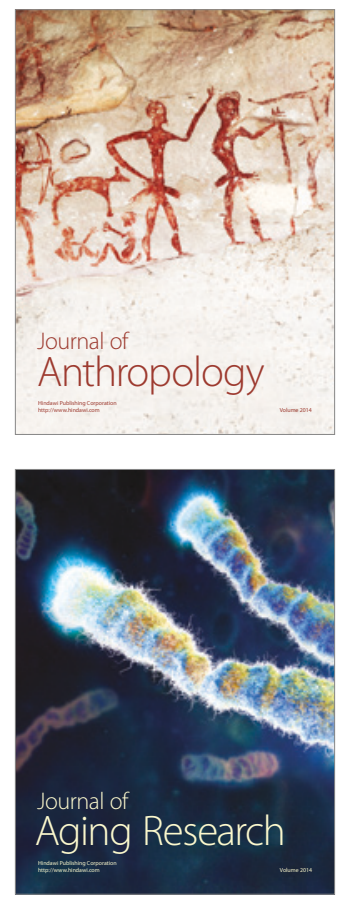
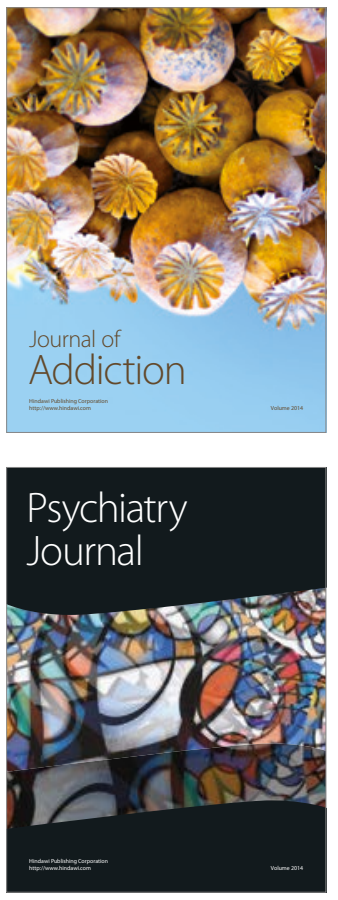

Child Development

Research

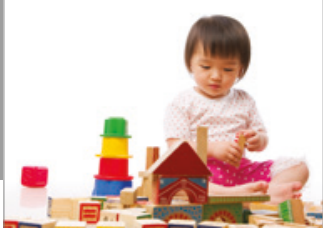

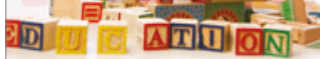
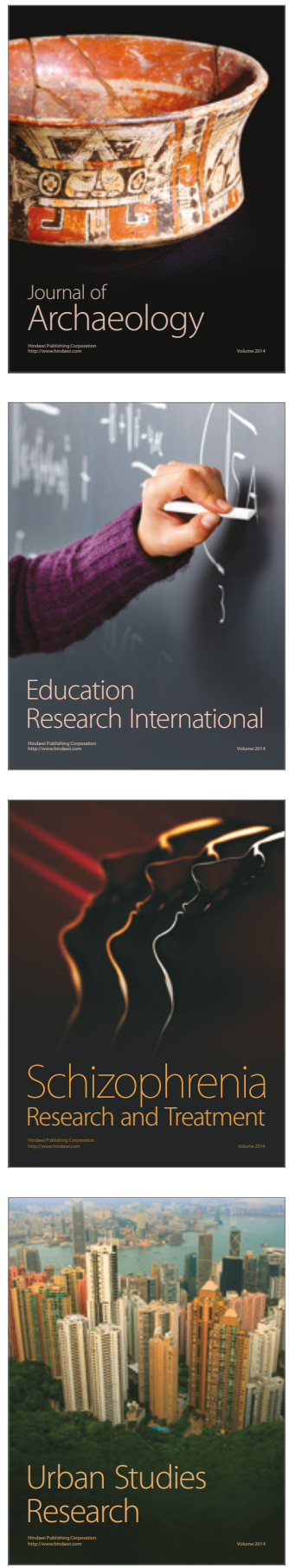